\title{
Primeira experiência de tireoidectomia endoscópica transoral por acesso vestibular (TOETVA) no Hospital Universitário Walter Cantídio: relato de dois casos
}

\section{First experience of transoral endoscopic thyroidectomy by vestibular approach (TOETVA) at University Hospital Walter Cantídio: a report of two cases}

Marcelo Emanuel Ericeira da Costa ${ }^{1}$. Wellington Alves Filho ${ }^{1,2}$. Francisco Januário Farias Pereira Filho ${ }^{1,2}$. Caio Fortier Silva ${ }^{1}$. Letícia Chaves Vieira Cunha ${ }^{1}$. Luis Alberto Albano Ferreira ${ }^{1,2}$.

1 Universidade Federal do Ceará (UFC), Fortaleza, Ceará, Brasil. 2 Hospital Universitário Walter Cantídio (HUWC), Fortaleza, Ceará, Brasil.

\section{RESUMO}

Objetivos: Relatar a experiência inicial com a tireoidectomia transoral endoscópica por abordagem vestibular (TOETVA) no Hospital Universitário Walter Cantídio - UFC. Metodologia: Relatamos o caso de duas pacientes submetidas à TOETVA no Serviço de Cirurgia de Cabeça e Pescoço do Hospital Universitário Walter Cantídio-UFC. As pacientes do estudo tinham 40 e 31 anos de idade, ASA I, com nódulo de 3,9 cm e 2,1 cm, respectivamente. Dados das pacientes, citologia, complicações, tempo cirúrgico, sintomas pós-operatórios e tempo de internação foram registrados. Resultados: Duas pacientes do sexo feminino foram submetidas a tireoidectomia parcial em maio de 2019. Suas respectivas idades eram 40 e 31 anos. Citologia: Bethesda II e Bethesda IV. O resultado histopatológico final confirmou benignidade. As duas pacientes queixaram-se de dor e hipoestesia no mento de baixa intensidade no pós-operatório. A internação de ambas as pacientes durou dois dias. Conclusões: A TOETVA é uma técnica cirúrgica relativamente nova, porém, segura e eficaz, com excelentes resultados estéticos, sendo uma excelente possibilidade de tratamento para pacientes selecionados.

Palavras-chave: Glândula tireoide. Tireoidectomia. Procedimentos cirúrgicos minimamente invasivos.

\section{ABSTRACT}

Objectives: Our main objective is to report the initial experience with endoscopic transoral vestibular approach thyroidectomy (TOETVA) at the Walter Cantídio University Hospital - UFC. Methodology: We report the cases of two patients undergoing TOETVA at the Head and Neck Surgery Service of the Walter Cantídio University Hospital-UFC. The patients were aged 40 and 31 years, ASA I, with single thyroid nodules measuring $3.9 \mathrm{~cm}$ and $2.1 \mathrm{~cm}$, respectively. Patient data, cytology, complications, surgical time, postoperative symptoms and length of hospital stay were recorded. Results: Two female patients underwent partial thyroidectomy in May 2019. Their respective ages were 39 and 31 years. The cytology reports regarding previous thin needle aspiration biopsy revealed Bethesda scores of II and IV, respectively. The final histopathological result confirmed benignity. Both patients complained of postoperative low-intensity pain and hypoesthesia. The hospitalization of both patients lasted two days. Conclusions: TOETVA is a relatively new, yet safe and effective, surgical technique with excellent aesthetic results. It is an excellent treatment possibility for selected patients.

Keywords: Thyroid gland. Thyroidectomy. Minimally invasive surgical procedures.

Autor correspondente: Marcelo Emanuel Ericeira da Costa, Avenida Sargento Hermínio Sampaio, 1415, Monte Castelo, Fortaleza, Ceará. CEP: 60326-515. Telefone: +5598 98103-3648. E-mail: marceloericeira@hotmail.com

Conflito de interesses: Não há qualquer conflito de interesses por parte de qualquer um dos autores.

Recebido em: 25 Nov 2019; Revisado em: 16 Fev 2020; Aceito em: 16 Fev 2020. 


\section{INTRODUÇÃO}

Desde a descrição da técnica cirúrgica de tireoidectomia em um trabalho científico do ano de 1883 por Kocher, fato que o levou a receber o Prêmio Nobel de Medicina no ano de 1909, houve poucas modificações em relação à técnica de tireoidectomia convencional realizada nos dias atuais que requer incisão transversal da pele ao longo do pescoço. ${ }^{1}$

Na década de 1980, técnicas cirúrgicas minimamente invasivas atraíram interesse em todas as especialidades cirúrgicas. ${ }^{2}$ Muitos cirurgiões estão desenvolvendo técnicas com o objetivo de alcançar melhor resultado cosmético em relação a cicatriz cervical, ${ }^{3}$ a exemplo de Miccoli que desenvolveu a técnica de tireoidectomia minimamente invasiva video-assistida com uma incisão cervical reduzida de $1,5 \mathrm{~cm} .{ }^{3}$ Mesmo com esses avanços, até mesmo pequenas cicatrizes no pescoço são altamente insatisfatórias para alguns indivíduos. ${ }^{2}$ Ikeda, em 2000, desenvolveu a técnica de tireoidectomia por acesso axilar em que a cicatriz deixada na axila estava completamente coberta pelo braço do paciente em sua posição natural. Essa técnica, no entanto, impedia uma abordagem bilateral no mesmo momento. ${ }^{2}$ Além das desvantagens específicas de cada técnica desenvolvida, essas causavam graves danos aos tecidos, muitas complicações, altas taxas de conversão em cirurgias abertas e dificuldades cirúrgicas devido à limitação de movimento.

Em 2015, Anuwong publicou um estudo com 60 casos operados na Tailândia utilizando a técnica de tireoidectomia endoscópica transoral por acesso vestibular (TOETVA), em que a incisão cervical não era mais necessária para a realização de tireoidectomia, visto que seria possível excisar a glândula através de incisão vestibular, não deixando a cicatriz cirúrgica visível na região cervical, com resultados comparáveis aos da tireoidectomia convencional por acesso cervical. ${ }^{4}$

Essa técnica requer instrumentos laparoscópicos convencionais e uma tesoura ultrassônica, causa menos danos aos tecidos, sendo mais segura e mais viável em comparação com as técnicas anteriores. ${ }^{4}$ A principal vantagem para os pacientes na escolha do TOETVA, em relação à cirurgia aberta, foi um excelente resultado cosmético, sem cicatriz visível na região cervical.

Este trabalho tem como objetivo relatar a experiência inicial da utilização da técnica cirúrgica de tireoidectomia endoscópica transoral por acesso vestibular (TOETVA) no Hospital Universitário Walter Cantídio-CE, discutindo as particularidades dessa inovação técnica. Este trabalho foi aprovado pelo comitê de ética em pesquisa do Hospital Universitário Walter Cantídio.

\section{RELATO DE CASO}

\section{Técnica cirúrgica}

O procedimento cirúrgico foi realizado sob anestesia geral com intubação orotraqueal, a paciente foi posicionada em decúbito dorsal horizontal com extensão cervical conforme a técnica de tireoidectomia convencional.

Ampicilina/sulbactan foi utilizado como antibiótico profilático pré-operatório. A cavidade oral foi higienizada com clorexidina aquosa $0,2 \%$.

Foram realizadas três incisões em vestíbulo oral, sendos estas: uma incisão de $1,5 \mathrm{~cm}$ em porção mediana do vestíbulo oral e duas incisões de $0,5 \mathrm{~cm}$ em posição lateral próximas ao vermelhão do lábio, ao nível dos caninos. Posteriormente uma pinça de Kelly foi utilizada para divulsionar os tecidos através destas incisões até o pescoço anterior, tangenciando a mandíbula (Figura 1 e Figura 2).

Foi alocado, pela incisão de $1,5 \mathrm{~cm}$, uma vela de HEGAR tamanho 8 e introduzido realizando dissecção às cegas abaixo da camada subplatismal até a região superior à fúrcula esternal.

Figura 1. Incisões no vestíbulo oral.

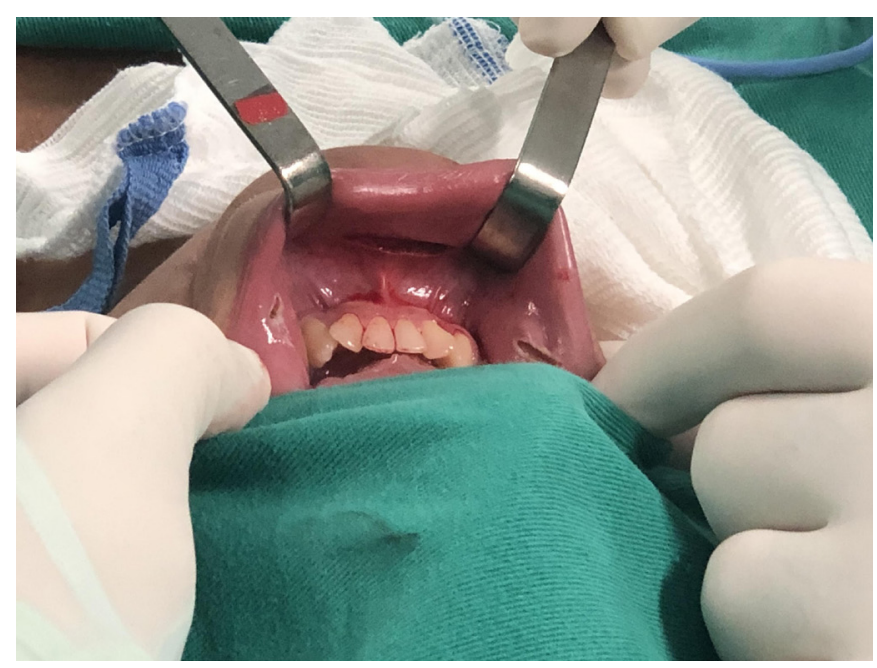

Figura 2. Incisões cirúrgicas suturadas.

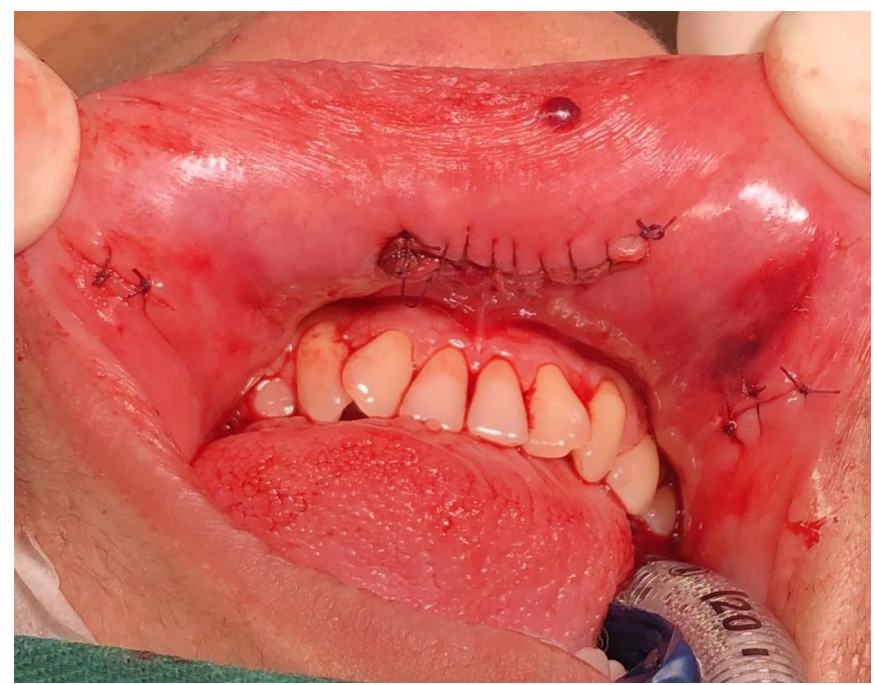


Foram alocados trocateres de videocirurgia de $10 \mathrm{~mm}$ na incisão mediana e dois trocateres de $5 \mathrm{~mm}$ em cada incisão lateral e instrumentos laparoscópicos convencionais foram introduzidos. Posteriormente foi realizado insuflação de $\mathrm{CO} 2$, pressão $6 \mathrm{mmHg}$ e fluxo $15 \mathrm{l} / \mathrm{min}$. A configuração da pressão de CO2 não deve ser superior a $6 \mathrm{mmHg}$, para evitar enfisema mediastinal.

Utilizando tesoura ultrassônica foi dissecado o tecido subplatismal. A linha Alba entre os músculos pré-tireoideanos foi evidenciada e realizada a secção com tesoura ultrassônica, sendo esta indispensável para essa cirurgia. Realizou-se a istmotomia. Posteriormente a musculatura pré-tireoideana ipsilateral ao lado da tireoidectomia foi retraída por sutura de suspensão externa (Figura 3).

Procedeu a secção do pedículo vascular (artéria e veia tireoidiana) superior do lobo tireoidiano que seria abordado, sendo posteriormente o polo tireoidiano levantado e foi iniciado a procura do nervo laríngeo recorrente e confirmado com monitorização de nervo.

A peça cirúrgica foi removida através de uma endobag pela incisão vestibular. Não foi utilizado dreno. Os músculos pré-tireoidianos não foram reaproximados.

As incisões na cavidade oral foram suturadas. Um curativo de compressão em região do mento foi colocado no pós-operatório imediato e orientado a troca diariamente (Figura 4).

\section{PRIMEIRO CASO}

Paciente do sexo feminino, 40 anos de idade, ASA I, assintomática. Ultrassonografia evidenciando nódulo de $3,4 \mathrm{~cm}$ em lobo esquerdo da tireoide. Este nódulo foi puncionado previamente à cirurgia. $\mathrm{O}$ resultado da punção aspirativa por agulha fina (PAAF) do nódulo foi Bethesda II (citologia benigna).

A paciente foi submetida a tireoidectomia parcial esquerda sob a técnica TOETVA. Ampicilina/sulbactan foi utilizado na indução anestésica. Iniciou cefazolina no pós-operatório. O tempo de cirurgia foi de 120 minutos, sem intercorrências. O nervo laríngeo recorrente foi confirmado com monitor de nervo laríngeo recorrente. Não houve lesão de nervo laríngeo recorrente.

Dieta oral branda foi iniciada no primeiro dia pós-operatório. Paciente recebeu alta no segundo dia pós-operatório. Foi prescrito amoxicilina/clavulanato para uso domiciliar por 7 dias.

A paciente relatou no pós-operatório dor moderada e hipoestesia leve na região do mento e apresentava equimose em região mentoniana e região cervical. Retornou para consulta ambulatorial com 1 semana, 1 mês, 2 meses e 4 meses relatando discreta sensação de hipoestesia em região mentoniana. Histopatológico evidenciou adenoma folicular.
Laringoscopia foi realizada no primeiro retorno ambulatorial com 7 dias e apresentava características normais. A paciente relata ter ficado muito satisfeita com o resultado estético.

Figura 3. Intraoperatório.

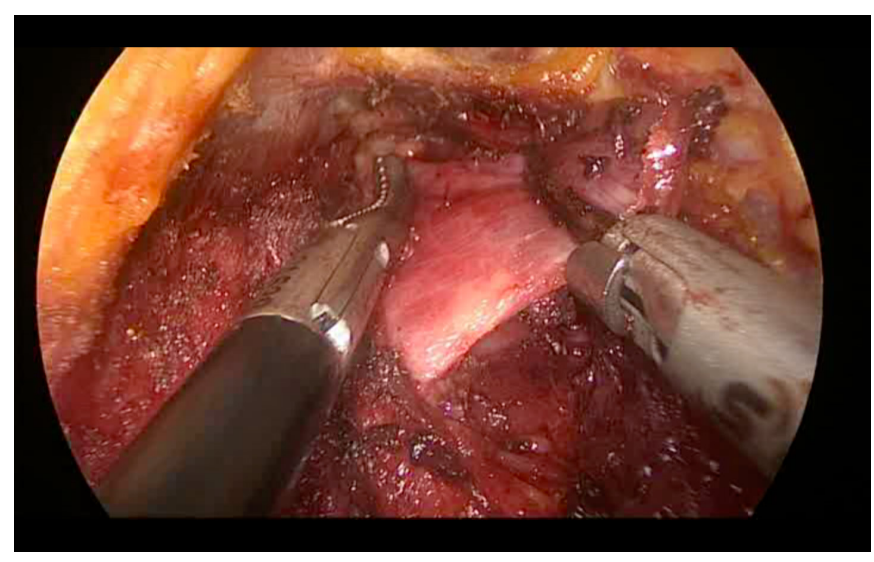

Figura 4. Pescoço no pós-operatório imediato.

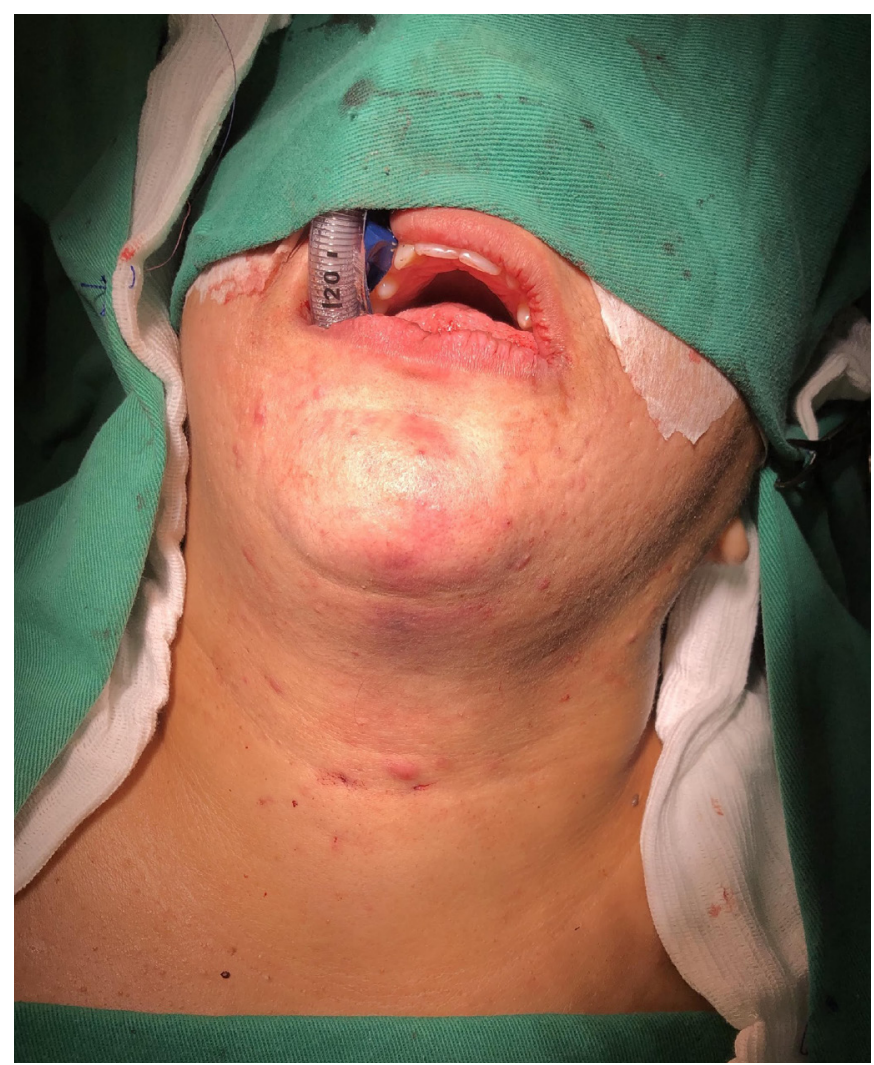

\section{SEGUNDO CASO}

Paciente do sexo feminino, 31 anos, ASA I, assintomática. Ultrassonografia evidenciando nódulo tireoideano de $2,1 \mathrm{~cm}$ terço médio e nódulo de $0,8 \mathrm{~cm}$ em terço inferior do lobo direito da tireoide. O exame também evidenciou nódulos infracentimétricos de $0,3 \mathrm{~cm}$ e $0,2 \mathrm{~cm}$ em lobo esquerdo. $\mathrm{O}$ 
nódulo maior do lobo direito foi puncionado previamente à cirurgia. $\mathrm{O}$ resultado da punção aspirativa por agulha fina (PAAF) do nódulo foi Bethesda IV (citologia suspeita para neoplasia folicular). A paciente foi submetida a tireoidectomia parcial direita sob a técnica TOETVA.

Ampicilina/sulbactan foi utilizado na indução anestésica e mantido no pós-operatório. O tempo de cirurgia foi de 180 minutos, sem complicações. O nervo laríngeo recorrente foi confirmado com monitor de nervo. Não houve lesão de nervo laríngeo recorrente.

Paciente relatou no pós-operatório dor submental e em região submandibular de moderada intensidade, hipoestesia leve na região do mento. Apresentava ainda equimose discreta em região cervical. Dieta oral branda foi iniciada no primeiro dia pós-operatório.

Paciente recebeu alta no segundo dia pós-operatório. Foi prescrito amoxicilina/clavulanato para uso domiciliar por 7 dias.

A paciente retornou para consulta ambulatorial com 2 semanas relatando ainda dor em mandíbula com melhora parcial ao uso de analgésicos e hipestesia em mento. No retorno ambulatorial de 1 mês relatou melhora dos sintomas e no retorno ambulatorial de 2 meses relatou leve parestesia de lábio inferior e região mentoniana. Histopatológico evidenciou adenoma folicular. Laringoscopia foi realizada no primeiro retorno ambulatorial com 14 dias e apresentava características normais. Relata ter ficado satisfeita com resultado estético (Figura 5).

Figura 5. Incisão cirúrgica 2 meses após a cirurgia.

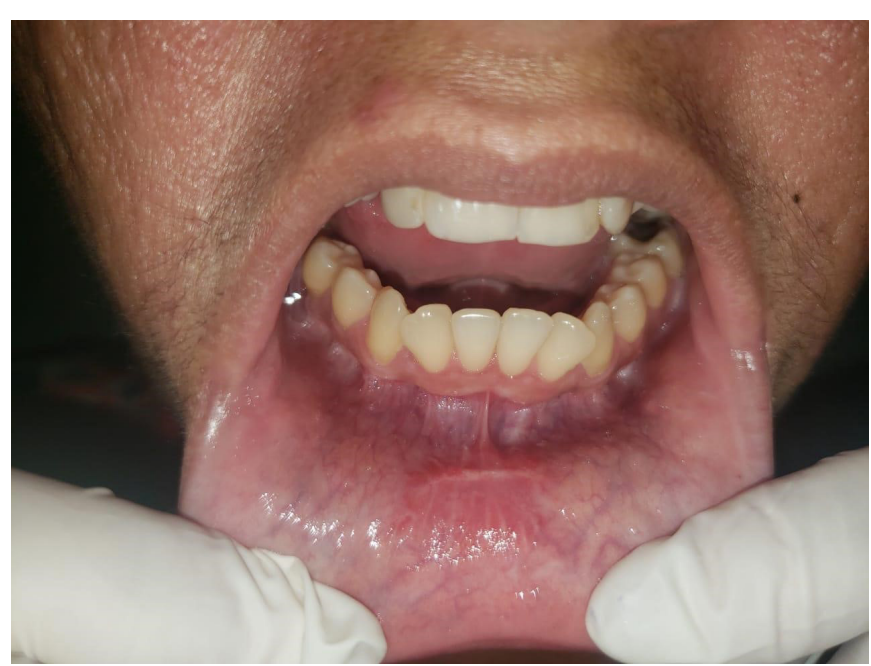

\section{REFERÊNCIAS}

1. Cernea CR, Brandão LG. Kocher e a história da tireoidectomia. Rev Bras Cir Cabeça Pescoço. 2008;37(4):240-3.

2. Ikeda Y, Takami H, Sasaki Y, Kan S, Niimi M. Endoscopic neck

\section{DISCUSSÃO}

A TOETVA é uma técnica muito nova realizada com instrumentos laparoscópicos convencionais. Apesar de amplamente adotada na Ásia, a experiência na Europa e na América ainda é pequena. ${ }^{5}$

Apesar da região anterior do pescoço favorecer a formação de uma cicatriz discreta, até mesmo pequenas cicatrizes no pescoço são altamente insatisfatórias para alguns indivíduos. ${ }^{2}$ A TOETVA elimina por completo qualquer preocupação com questões estéticas e, quando comparada a outras técnicas endoscópicas, possui algumas vantagens como: menor área de dissecção, possibilita o acesso aos dois lobos da glândula através da mesma incisão, o equipamento cirúrgico é o mesmo utilizado em cirurgia videolaparoscópica e os custos são menores quando comparados com a cirurgia robótica. ${ }^{5}$

Os critérios para o paciente ser submetido à técnica TOETVA: pacientes com diâmetro da glândula tireóide não superior a 10 cm que possui (1) nódulos benignos, como cisto da tireoide, bócio nodular único ou bócio multinodular, (2) neoplasia folicular, (3) doença de Graves ou (4) um microcarcinoma papilifero da tireoide sem evidência de metástases. ${ }^{4}$

A taxa de infecção é muito baixa e a literatura não relatou nenhum caso de infecção associada à técnica transvestibular até o presente momento.

O uso de um bisturi harmônico aumenta os custos gerais da cirurgia, ${ }^{3}$ além de não estar disponível em todos os hospitais públicos, o que seria um fator limitante para a realização da cirurgia em maior número.

Tireoides de grande tamanho ou consistência mais dura precisam ser fraturadas em pedaços menores antes da remoção, o que poderia comprometer a adequada avaliação anatomopatológica.

Foram realizadas no Hospital Universitário Walter Cantidio, de maio a novembro de 2019, cinco tireoidectomias por acesso transvestibular endoscópico, sendo quatro lobectomias direita e uma lobectomia esquerda. A principal queixa no acompanhamento ambulatorial é a hipoestesia em região do mento, é descrito na literatura a permanência dessa queixa até, em média, 6 meses após o procedimento cirúrgico. ${ }^{6}$

Em nossa experiência, observamos que a TOETVA, apesar de ser uma técnica cirúrgica relativamente nova, é segura e eficaz e proporciona excelentes resultados estéticos, sendo uma excelente possibilidade de tratamento para pacientes selecionados.

surgery by the axillary approach. J Am Coll Surg. 2000;191(3):336-40.

3. Miccoli P, Berti P, Ambrosini CE. Perspectives and lessons learned after a decade of minimally invasive video- 
assisted thyroidectomy. ORL J Otorhinolaryngol Relat Spec. 2008;70(5):282-6.

4. Anuwong A. Transoral endoscopic thyroidectomy vestibular approach: a series of the first 60 human cases. World J Surg. 2016;40(3):491-7.

5.Tesseroli MA, Spagnol M, Sanabria A. Transoral endoscopic thyroidectomy by vestibular approach (TOETVA): initial experience in Brazil. Rev Col Bras Cir. 2018;14;45(5):e1951.

6.Nakajo A, Arima H, Hirata M, Mizoguchi T, Kijima Y, Mori S, et al. Trans-oral video- assisted neck surgery (TOVANS). A new transoral technique of endoscopic thyroidectomy with gasless premandible approach. Surg Endosc. 2013;27(4):1105-10.

7.Miccoli P, Pinchera A, Materazzi G, Biagini A, Berti P, Faviana P, et al. Surgical treatment of low- and intermediate-risk papillary thyroid cancer with minimally invasive video-assisted thyroidectomy. J Clin Endocrinol Metab. 2009;94(5):1618-22.

8.Kang SW, Lee SC, Lee SH, Lee KY, Jeong JJ, Lee YS, et al. Robotic thyroid surgery using a gasless, transaxillary approach and the da Vinci S system: the operative outcomes of 338 consecutive patients. Surgery. 2009;146(6):1048-55.

9.Adam MA, Speicher P, Pura J, Dinan MA, Reed SD, Roman SA, et al. Robotic thyroidectomy for cancer in the US: patterns of use and short-term outcomes. Ann Surg Oncol. 2014;21(12):3859-64.

10.Chai YJ, Kim HY, Kim HK, Jun SH, Dionigi G, Anuwong A, et al. Comparative analysis of 2 robotic thyroidectomy procedures: Transoral versus bilateral axillo-breast approach. Head Neck. 2018;40(50):886-92.

11.Pan JH, Zhou H, Zhao XX, Ding H, Wei L, Qin L, et al. Robotic thyroidectomy versus conventional open thyroidectomy for thyroid cancer: a systematic review and meta-analysis. Surg Endosc. 2017;31:3985-4001.

12. Miccoli P, Bellantone R, Mourad M, Walz M, Raffaelli M, Berti P. Minimally invasive video-assisted thyroidectomy: multiinstitutional experience. World J Surg. 2002;26(8):972-5.

13.Ikeda Y, Takami H, Niimi M, Kan S, Sasaki Y, Takayama J. Endoscopic thyroidectomy and parathyroidectomy by the axillary approach: a preliminary report. Surg Endosc. 2002;16(1):92-5.

\section{Como citar:}

Costa ME, Alves W Filho, Pereira FJ Filho, Silva CF, Cunha LC, Ferreira LA. Primeira experiência de tireoidectomia endoscópica transoral por acesso vestibular (TOETVA) no Hospital Universitário Walter Cantídio: relato de dois casos. Rev Med UFC. 2020 jul-set;60(3):51-55. 